The following scientific article was officially published in the proceedings of the international conference SPIE Medical Imaging 2012: Computer-Aided Diagnosis (Vol. 8315). This article's citation is as follows:

Adankon, Mathias M., Jean Dansereau, Stefan Parent, Hubert Labelle, and Farida Cheriet. "Scoliosis curve type classification from 3D trunk image." In SPIE Medical Imaging, (2012) pp. 831514--831514-7.

doi: http://dx.doi.org/10.1117/12.911335

The manuscript, as submitted by the first author, is reproduced in the following pages.

(C) 2012 Society of Photo-Optical Instrumentation Engineers. One print or electronic copy may be made for personal use only. Systematic reproduction and distribution, duplication of any material in this paper for a fee or for commercial purposes, or modification of the content of the paper are prohibited. 


\title{
Scoliosis curve type classification using kernel machine from 3D trunk image
}

\author{
Mathias M. Adankon ${ }^{a, b}$, Jean Dansereau ${ }^{a, b}$, Stefan Parent ${ }^{b}$, Hubert Labelle ${ }^{b}$ and Farida Cheriet ${ }^{a, b}$ \\ ${ }^{a}$ Ecole Polytechnique de Montreal, Montreal, Canada; \\ ${ }^{b}$ Sainte-Justine Hospital Research Center, Montreal, Canada.
}

\begin{abstract}
Adolescent idiopathic scoliosis (AIS) is a deformity of the spine manifested by asymmetry and deformities of the external surface of the trunk. Classification of scoliosis deformities according to curve type is used to plan management of scoliosis patients. Currently, scoliosis curve type is determined based on X-ray exam. However, cumulative exposure to X-rays radiation significantly increases the risk for certain cancer. In this paper, we propose a robust system that can classify the scoliosis curve type from non invasive acquisition of 3D trunk surface of the patients. The 3D image of the trunk is divided into patches and local geometric descriptors characterizing the surface of the back are computed from each patch and forming the features. We perform the reduction of the dimensionality by using Principal Component Analysis and 53 components were retained. In this work a multi-class classifier is built with Least-squares support vector machine (LS-SVM) which is a kernel classifier. For this study, a new kernel was designed in order to achieve a robust classifier in comparison with polynomial and Gaussian kernel. The proposed system was validated using data of 103 patients with different scoliosis curve types diagnosed and classified by an orthopedic surgeon from the X-ray images. The average rate of successful classification was $93.3 \%$ with a better rate of prediction for the major thoracic and lumbar/thoracolumbar types.
\end{abstract}

Keywords: Scoliosis, Classifier design, 3D trunk image modeling.

\section{INTRODUCTION}

Adolescent idiopathic scoliosis (AIS) is a deformity of the spine manifested by asymmetry and deformities of the external surface of the trunk. It consists of a complex curvature in the three-dimensional space: inclination in the frontal plane, rotation of vertebrae in the horizontal plane and inversion of the curves in the sagittal plane. This pathology is often visible, but it may pass as unnoticed during its development for years. There are a wide variety of deformities of the spine, however, a classification of major curve types is possible. The classification of different deformities is used to group similar curves in order to define an appropriate treatment strategy.

Currently, X-ray exam is performed in order to determine the scoliosis curve type and its severity. The scoliosis X-ray includes the entire spine image, thoracic part (upper back) and the lumbar part (lower back). Scoliosis curves are classified by their location in the spine and the magnitude of the curve. While 1 in 25 people have mild scoliosis deformities, only 1 in 200 adolescents have deformities that progress to require either bracing or surgical treatment. Since there is as yet no reliable way to predict which deformities will progress, these suspected patients are monitored with a series of X-rays acquired semi-annually during rapid adolescent growth. However, cumulative exposure to X-rays radiation significantly increases the risk for certain cancer (1).

During the last 30 years, many optical non invasive surface measurement systems have been developed based on a 3D reconstruction of the back or of the whole trunk. The main goal of the non invasive approach is to reduce the radiographic evaluation while providing reliable information to the clinician. Many methods have been developed for characterizing scoliosis torso shape deformity $(2 ; 3)$.

\footnotetext{
Mathias Adankon, Jean Dansereau and Farida Cheriet are with Ecole Polytechnique de Montreal, Montreal, Canada. (email: mathias-mahouzonsou.adankon@polymtl.ca, jean.dansereau@polymtl.ca, farida.cheriet@ polymtl.ca)

Hubert Labelle and Stefan Parent are with Sainte-Justine Hospital Research Center, Montreal, Canada (email: hubert.labelle@ rechercheste-justine.qc.ca, stefan.parent@umontreal.ca).

This work was supported by the GRSTB, the MENTOR program and the CIHR (Canadian Institutes of Health Research).
} 
However, to our knowledge, studies on non invasive prediction of scoliosis type or severity using machine learning method are limited $(4 ; 5 ; 6 ; 7)$. In the first work (4), an artificial neural network combined with genetic algorithm is used in order to estimate the Cobb angle. The results reveal that the system predict the Cobb angle within 5 degrees in 65 percent on the test. The major problem that occurs in this study is the over-fitting because the machine gives a good result on training samples but has poor predictive performance during testing phase. In (5), a spinal curve was predicted based on the trunk surface image. This study has attempted to find a nonlinear correlation between the interior and the exterior geometry. An array of support vector regression machines is built to predict the spinal coefficients which constitute comprehensive features for spinal curve description. However, the results are moderate with $70-82 \%$ of correct prediction. In (6), the authors proposed a prediction system where the subjects were classified into 3 severity groups (mild, moderate, severe) using 3D back shape image combined with other indicators like sex, age, etc. And, their system achieved 69-85\% accuracy in testing. In contrast, Lama et al.(7) have proposed for the first time a prediction of the scoliosis curve type using only the trunk 3D image. In this work, the authors have considered the Lenke classification which uses also bending test; thus, the system gave a moderate result, $72 \%$ of correct classification.

The previous studies have obtained moderate results partly because of the small number of the available data which is a critical problem in medicine research field. In fact, the performance of many learning algorithms, as artificial neural network, are very dependent on the number of training sample. Thus, in this paper, we propose an improved non invasive classification system for scoliosis curve type (thoracic major curve, thoracolumbar major curve, lumbar major curves and double major curves) using a kernel classifier, Least Squares Support Vector Machine (LS-SVM) which has excellent capacity of generalization (8). In this study, a new kernel function is designed in order to empower the classifier performance. The 3D image of the back surface is divided into patches and local geometric descriptors are computed from each patch forming the features and classification is performed using a combination of LS-SVM classifiers. The proposed system is illustrated in Figure 1.

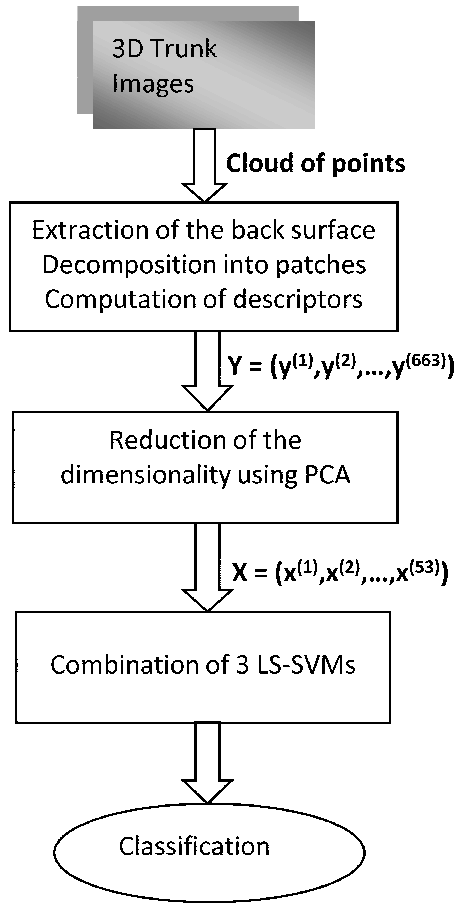

Figure 1. General overview of the Classification system. 


\section{MATERIALS AND METHODS}

\subsection{Data acquisition}

Since many years, the acquisition of the trunk surface topography is part of the routine evaluation of scoliosis patients at Sainte-Justine University Hospital Center (SJUHC) in Montreal (Canada). The acquisition system is composed of four optical digitizers (CREAFORM, Montreal, Canada). Each optical digitizer contains one color CCD camera and a structured light projector. The acquisition process of each digitizer is as follows. Four fringe patterns, obtained by phase-shifting technique, are successively projected onto the surface. Based on the four resulting images and triangulation technique, the system computes the depth of each surface point relative to the reference plane. A fifth image, with no fringes, acquires the texture of the surface which is then mapped onto the 3D reconstruction.

For the reconstruction of the whole trunk, four scanners are placed around the patient (on the front, on the back and at $\pm 60^{\circ}$ laterally in front of the patient). Each digitizer reconstructs a portion of the trunk. During the acquisition, approximately 4 seconds, the patient stands still in the upright position with the arms slightly abducted in order not to obstruct the lateral scanners fields of view. Based on a multi-head calibration of the system that computes the rigid transformations between the digitizers, the 4 portions of the trunk are registered and merged using EM software. The resulting mesh is constituted of 40,000 to 70,000 nodes, depending on the size of the patient. The accuracy of this system was evaluated in (9), using markers placed on a mannequin whose coordinates were previously recorded by a computer measuring machine. The results showed a reconstruction accuracy of $1.4 \mathrm{~mm}$ over the whole torso and of $0.56 \mathrm{~mm}$ over the back.

\subsection{Features extraction}

The 3D trunk image is divided into $h$ equal parts using horizontal planes as shown in Figure 2-a. Using cylindrical coordinates $(\rho, \phi, z)$, each transversal slice is divided in $n$ patches by varying the azimuth $\phi$ (see Figure 2-b). Thus, the whole 3D image is split up into $n * h$ patches and the geometric descriptors are calculated from each patch. First, we approximate each patch (piece surface) by a plane and the normal vector $\left(v_{x}, v_{y}, v_{z}\right)$ of this plane is kept as a descriptor. Then, from each 3D trunk image, we compute $3 n h$ descriptors.

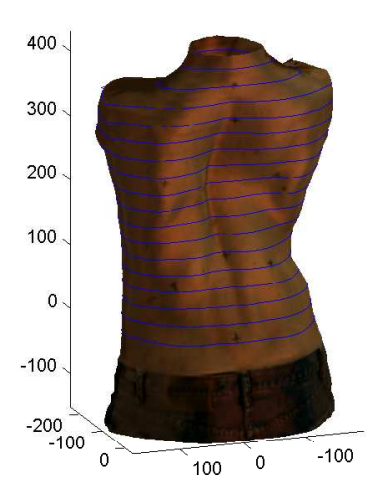

(a)

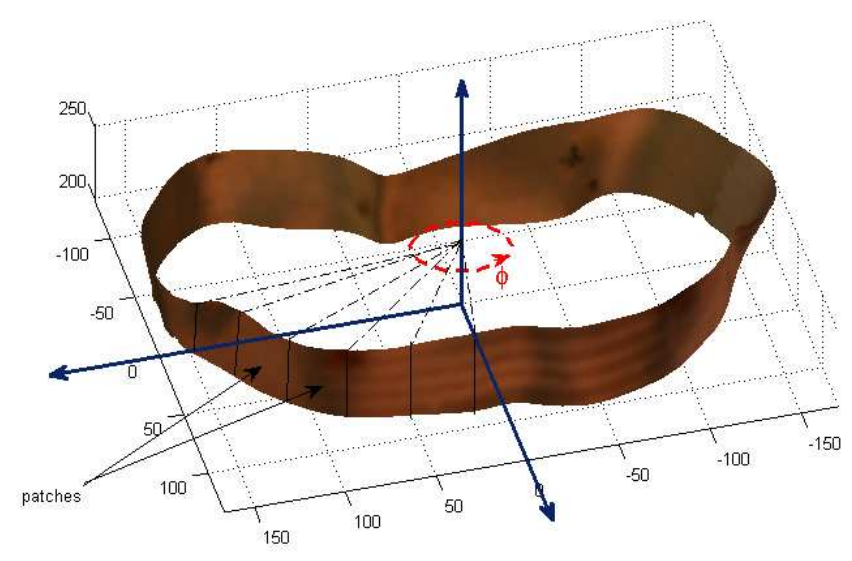

(b)

Figure 2. Features extraction: (a)Decomposition of trunk into slices. (b) Decomposition of transversal slice into patches.

In this study, we set $h=17$ and $n=13$; and we consider only the back by fixing $\phi$ between 0 and $\pi$. We divided the trunk in two regions: thoracic region and lumbar region. The first 10 slices form the thoracic region and the rest constitutes the lumbar region*. Thus, we obtained respectively 390 and 273 descriptors for thoracic and lumbar regions. In sum we have 663 descriptors for each 3D image.

\footnotetext{
${ }^{*}$ The relationship between the size of thoracic and lumbar regions is approximatively $\mathrm{T} / \mathrm{L}=1.4$ according to Jean Cruveilhier, Anatomie descriptive, Volume 1, 1837, page 49.
} 
In general, building a classifier based on statistical methods with an important number of features is not recommended because working in high-dimensional space involves the curse of dimensionality problem. Thus, for this work, we apply Principal Component Analysis (PCA), which is an orthogonal linear transformation, in order to reduce the dimensionality of the data. The number of components is selected based on the rate $(90 \%)$ of the total variance in the observed variables retained by the selected principal components: 28 components for the thoracic region and 25 components for the lumbar region (see Figure 3). Thus, we represent each trunk by 53 features.

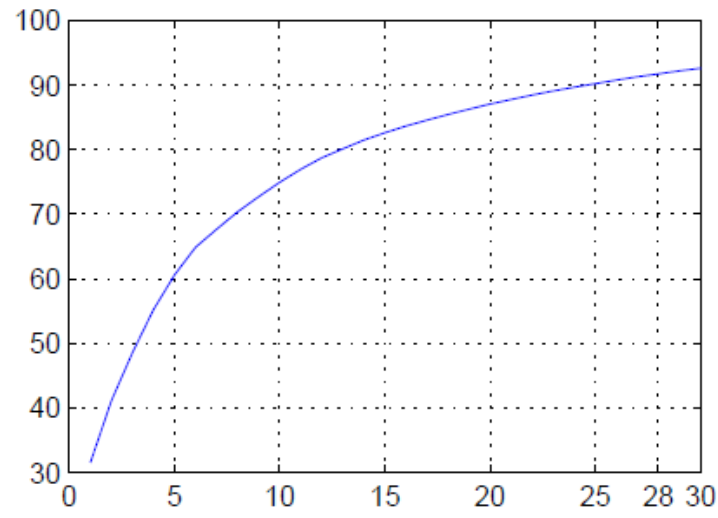

(a)

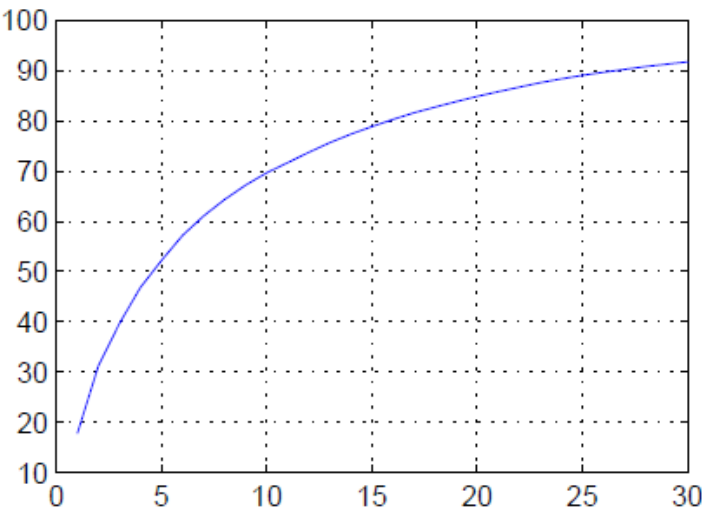

(b)

Figure 3. Rate of total variance in the observed variables w.r.t. the number of principal components (a) Thoracic region; (b) Lumbar region.

\subsection{Classifier design}

As classifier, we used Least Squares Support Vector Machine (LS-SVM) which is based on the margin maximization principle (8). Considering a binary classification problem involving a dataset $\left\{\left(x_{1}, y_{1}\right), \ldots,\left(x_{\ell}, y_{\ell}\right)\right\}$ with $x_{i} \in \mathcal{R}^{d}$ and $y_{i} \in\{-1,1\}$; nonlinear LS-SVM classifiers use the kernel trick to produce nonlinear boundaries. The decision function given by an LS-SVM is :

$$
f(x)=\operatorname{sign}\left[w^{\prime} \phi(x)+b\right]
$$

where $w$ and $b$ are found by resolving the following optimization problem which expresses the maximization of the margin $2 /\|w\|$ and the minimization of the training error:

$$
\begin{array}{r}
\min _{w, b, \xi} \frac{1}{2} w^{\prime} w+\frac{1}{2} \gamma \sum_{i=1}^{\ell} \xi_{i}^{2} \\
\text { subject to : } \xi_{i}=y_{i}-\left[w^{\prime} \phi\left(x_{i}\right)+b\right] \quad \forall i=1, \ldots, \ell
\end{array}
$$

LS-SVM is a variant of the standard SVM where the original SVM formulation is modified at two points (10). First, the inequality constraints with the slack variable $\xi_{i}$ are replaced by equality constraints. Second, a squared loss function is considered in the objective function. These two essential modifications simplify the problem, which becomes a linear system.

The Lagrangian of problem (2) is expressed by :

$$
\mathcal{L}=\frac{1}{2} w^{\prime} w+\frac{1}{2} \gamma \sum_{i=1}^{\ell} \xi_{i}^{2}-\sum_{i=1}^{\ell} \alpha_{i}\left\{y_{i}-\left[w^{\prime} \phi(x)+b\right]-\xi_{i}\right\}
$$

where $\alpha_{i}$ are Lagrange multipliers, which can be positive or negative because of equality constraints. 
The system arising from the Karush-Kuhn-Tucker conditions is linear, and the system of linear equations is expressed in the following matrix form:

$$
\left(\begin{array}{cc}
K+\gamma^{-1} I & \overrightarrow{1}^{\prime} \\
\overrightarrow{1} & 0
\end{array}\right)\left(\begin{array}{c}
\alpha \\
b
\end{array}\right)=\left(\begin{array}{c}
Y \\
0
\end{array}\right)
$$

where $K_{i j}=\phi\left(x_{i}\right) . \phi\left(x_{j}\right) ; Y=\left(y_{1}, \ldots, y_{\ell}\right)^{\prime} ; \alpha=\left(\alpha_{1}, \ldots, \alpha_{\ell}\right)^{\prime} ;$ and $\overrightarrow{1}=(1, \ldots, 1)$

\subsubsection{Design of a specific kernel}

The idea behind kernels is to map training data nonlinearly into a higher-dimensional feature space via a mapping function $\Phi$ and to construct a separating hyperplane which maximizes the margin $(11 ; 12)$. The construction of the linear decision surface in this feature space only requires the evaluation of dot products $\phi\left(x_{i}\right) \cdot \phi\left(x_{j}\right)=k\left(x_{i}, x_{j}\right)$, where the application $k: \mathcal{R}^{d} \times \mathcal{R}^{d} \rightarrow \mathcal{R}$ is called the kernel function. Using the kernel function, the decision function of SVM/LS-SVM is expressed in the following form: $f(x)=b+\sum_{i=1} \alpha_{i} k\left(x, x_{i}\right)$.

When we consider LS-SVM like other kernel classifiers, the choice of the kernel corresponds to choosing a function space for learning. The kernel determines the functional form of all possible solutions. Thus, the choice of the kernel is very important to construct a good machine. So, in order to obtain a good performance of the LS-SVM classifier, we need first to design or choose a type of kernel and then to optimize the hyperparameters for improving the capacity of generalization (13). In our context, we design a new kernel derivative from the Gaussian kernel where the features for the thoracic region and those for the lumbar region are weighted differently.

Considering two examples $x=\left(x_{(1)}, \ldots, x_{(T)}, x_{(T+1)}, \ldots, x_{(T+L)}\right)$ and $y=\left(y_{(1)}, \ldots, y_{(T)}, y_{(T+1)}, \ldots, y_{(T+L)}\right)$ where each element is formed by $T$ features obtained from the thoracic region and $L$ features from the lumbar region, we define our kernel function by:

$$
k(x, y)=\exp \left[-a \sum_{i=1}^{T}\left(x_{(i)}-y_{(i)}\right)^{2}-b \sum_{j=T+1}^{T+L}\left(x_{(j)}-y_{(j)}\right)^{2}\right]
$$

where $a>0$ and $b>0$ represent respectively the width of the kernel for the thoracic and lumbar regions.

\subsubsection{Classification Algorithm}

LS-SVM like classical SVM was designed first for two-class problem where the labels of the first class samples are assigned to +1 and the labels of the second class are assigned to -1 . Concerning multi-class problem, the classification task is divided in several two-class problems with adequate combination at the end. For our multi-class problem, we used one-against-all technique with rejection when all the LS-SVM outputs are negative. Thus, 3 LS-SVMs are trained, each separating one class from the others. During the test phase, the label is found by the following equation:

$$
y=\underset{i}{\operatorname{argmax}}\left[w_{i}^{\prime} \phi(x)+b_{i}\right]
$$

where $w_{i}$ and $b_{i}$ are the corresponding parameters of the LS-SVM trained for separating the samples of the class $i$ from the others.

\subsection{Validation}

Our classification system is validated on the dataset of 3D torso images of 103 patients with adolescent idiopathic scoliosis. The 3D trunk image and the radiography acquisitions have been done at the same visit for each patient. Based on the common clinical classification, the scoliosis curve type -thoracic major curves, thoracolumbar major curves, lumbar major curves and double major curves- was determined by an orthopedic surgeon from the X-ray images. The number of lumbar major curves being too small, we mixed them with the thoracolumbar major curves since these two types are nearly similar. The repartition of samples in these different classes is shown in Table 1.

Considering the size of the dataset $(\mathrm{N}=103)$ and the number of classes, we choose "leave-one-out" cross-validation for estimating the performance of our classification system. We validate the proposed system on the previous dataset and 
we make comparison with different kernel functions. For each system, the overall correct classification rate and correct classification per class are computed and the results are reported in the following section.

Table 1. Distribution of the patients among the classes

\begin{tabular}{lcc}
\hline Classes & Curve type & \# patients \\
\hline Class 1 & Thoracic major & 46 \\
Class 2 & Double major & 25 \\
Class 3 & Lumbar/thoracolumbar major & 32 \\
\hline Total & & 103 \\
\hline
\end{tabular}

\section{RESULTS AND DISCUSSION}

We perform the LOO cross-validation procedure through the 103 samples with our classification system which gives $93.3 \%$ of correct prediction. This result is interesting and shows that it is possible to predict the scoliosis curve type using only 3D back surface image of the patients. Also, the result confirms that the selected features extract the relevant information from the 3D back surface image for a scoliosis curve type classification task.

In Table 2, we report the correct prediction per class. Considering these results (last column), it is remarkable that the prediction of double major curve is the most difficult task. Further analysis of the result shows that almost the misclassification of double major curve samples are predicted as thoracic major curve. Thus, the major confusion of the classifier is produced between the double major curve and thoracic major curve. In fact, we know that it is also very difficult to an expert to separate these two groups using radiographs of the spine when the main curvature of the double major curve is thoracic. Thus, the clinicians in addition to the difference of the Cobb angle, develop the classification based on the bending test. On the other hand, the moderate predictive capacity of our classification system for class 2 comes also from the small number of the patients who have double major curve in the cohort.

Table 2. Classification rate per class

\begin{tabular}{lccc}
\hline Curve type & Polynomial kernel & Gaussian kernel & Proposed system \\
\hline Thoracic major & $90.7 \%$ & $95.3 \%$ & $95.3 \%$ \\
Double major & $83.3 \%$ & $81.25 \%$ & $\mathbf{8 4 . 2 1 \%}$ \\
Lumbar/thoracolumbar major & $93.5 \%$ & $96.4 \%$ & $96.4 \%$ \\
\hline Overall & $90.2 \%$ & $93.0 \%$ & $93.3 \%$ \\
\hline
\end{tabular}

In order to quantify the impact of our kernel with respect to the standard Gaussian kernel, we evaluate our system using other kernel functions: polynomial kernel and Gaussian kernel. We used grid search method to select the optimal value of the kernel parameters. The results are summarized in the table 2.

First, we note that the Gaussian kernel and the proposed kernel outperform the polynomial kernel. Since the proposed kernel is built from the Gaussian kernel, we can conclude that the Gaussian kernel is more suitable than the polynomial kernel for this classification task.

Second, with a designed kernel the recognition rate within the class 2, double major curve, was improved from $81.25 \%$ to $84.21 \%$. Thus, the overall recognition rate increased also. The results demonstrate that the proposed kernel performs better than the Gaussian kernel when we consider the classification of the scoliosis patients who have double major curve while keeping the good results achieved for class 1 and 3.

\section{CONCLUSION}

In this work, we proposed to build a system which is able to classify the scoliosis curve type using 3D trunk images which are obtained from non invasive acquisitions. Our system is validated on 103 patients and we obtained promising 
results. This shows that it is possible to find a relationship between the internal deformity and the torso shape deformity in scoliosis with machine learning methods. Also, the results indicate that relevant features based on geometric approach combined with statistical techniques can be extracted from 3D torso image in order to built a classification system with a high capacity.

However, we note that the classification capacity of our system for the double major curve could be ameliorated. Then, we can improve this part of our system by including the bending test descriptors. We expect that further work in this direction will yield better results and will improve the proposed system.

\section{References}

[1] Doody, M. M., Lonstein, J. E., Stovall, M., Hacker, D. G., Luckyanov, N., and Land, C. E., "Breast cancer mortality after diagnostic radiography - findings from the us scoliosis cohort study," Spine 25, 2052-2063 (Aug 2000).

[2] Jaremko, J. L., Poncet, P., Ronsky, J., Harder, J., Dansereau, J., Labelle, H., and Zernicke, R. F., "Estimation of spinal deformity in scoliosis from torso surface cross sections.," Spine (Phila Pa 1976) 26(14), 1583-91 (2001).

[3] Ajemba, P., Durdle, N., and Raso, V., "Characterizing torso shape deformity in scoliosis using structured splines models," Biomedical Engineering, IEEE Transactions on 56, 1652 -1662 (june 2009).

[4] Jaremko, J. L., [Estimation of Scoliosis Severity from the Torso Surface by Neural Networks], Ph.D. Thesis, Dept. Biomed. Eng., Univ. Calgary (2003).

[5] Bergeron, C., Cheriet, F., Ronsky, J., Zernicke, R., and Labelle, H., "Prediction of anterior scoliotic spinal curve from trunk surface using support vector regression," Eng. Appl. of AI 18(8), 973-983 (2005).

[6] Ramirez, L., Durdle, N., Raso, V., and Hill, D., "A support vector machines classifier to assess the severity of idiopathic scoliosis from surface topography," Information Technology in Biomedicine, IEEE Transactions on 10(1), $84-91$ (2006).

[7] Seoud, L., Adankon, M., Labelle, H., Dansereau, J., and Cheriet, F., "Prediction of scoliosis curve type based on the analysis of trunk surface topography," in [Biomedical Imaging: From Nano to Macro, 2010 IEEE International Symposium on ], $408-411$ (apr. 2010).

[8] Suykens, J. A. K., Van Gestel, T., De Brabanter, J., De Moor, B., and Vandewalle, J., [Least Squares Support Vector Machines. ], World Scientific, Singapore (2002).

[9] Pazos, V., Cheriet, F., Song, L., Labelle, H., and Dansereau, J., "Accuracy assessment of human trunk surface 3d reconstructions from an optical digitising system," Medical and Biological Engineering and Computing 43(1), 11-15 (2005).

[10] Adankon, M. M. and Cheriet, M., "Support vector machine," in [Encyclopedia of Biometrics], 1303-1308, Springer, US (2009).

[11] Boser, B. E., Guyon, I., and Vapnik, V., "A training algorithm for optimal margin classifiers," in [Computational Learing Theory], 144-152 (1992).

[12] Cristianini, N. and Shawe-Taylor, J., [An Introduction to Support Vector Machines], Cambridge University Press (2000).

[13] Adankon, M. M. and Cheriet, M., "Model selection for the ls-svm. application to handwriting recognition," Pattern Recognition 42(12), 3264-3270 (2009). 